Federico Ferreres • Carla Sousa •

Vendula Vrchovská • Patrícia Valentão •

José A. Pereira · Rosa M. Seabra • Paula B. Andrade

\title{
Chemical composition and antioxidant activity of tronchuda cabbage internal leaves
}

Received: 26 April 2005 / Revised: 21 June 2005 / Accepted: 1 July 2005 / Published online: 13 October 2005

(C) Springer-Verlag 2005

\begin{abstract}
A phytochemical study was undertaken on the internal leaves of tronchuda cabbage (Brassica oleracea L. var. costata DC). Seventeen phenolic compounds were characterized and quantified by reversed-phase HPLCDAD-ESI-MS ${ }^{n}$ and HPLC/DAD, respectively: quercetin 3$O$-sophoroside-7- $O$-glucoside, 3- $p$-coumaroylquinic acid, kaempferol 3-O-sophoroside-7-O-glucoside, kaempferol 3-O-(caffeoyl)-sophoroside-7- $O$-glucoside, sinapoyl glucoside acid, kaempferol 3-O-(sinapoyl)-sophoroside-7- $O$ glucoside, kaempferol 3-O-(feruloyl)-sophoroside-7-Oglucoside, kaempferol 3-O-( $p$-coumaroyl)-sophoroside7-O-glucoside, 4- $p$-coumaroylquinic acid, sinapic acid, kaempferol 3-O-sophoroside, 3 isomeric forms of 1,2disinapoylgentiobiose, 1-sinapoyl-2-feruloylgentiobiose, 1,2,2-trisinapoylgentiobiose and 1,2'-disinapoyl-2-feruloylgentiobiose. Seven organic acids (aconitic, citric, ascorbic, malic, quinic, shikimic and fumaric acids) were also identified and quantified. The hot water extract of tronchuda cabbage internal leaves was investigated for its
\end{abstract}

F. Ferreres

Research Group on Quality, Safety and Bioactivity of Plant Foods, Department of Food Science and Technology, CEBAS (CSIC), Campus University Espinardo,

P.O. Box 164, 30100 Murcia, Spain

C. Sousa $\cdot$ P. Valentão $\cdot$ R. M. Seabra $\cdot$ P. B. Andrade $(\square)$ REQUIMTE/ Serviço de Farmacognosia, Faculdade de Farmácia, Universidade do Porto,

R. Aníbal Cunha, 164,

4050-047 Porto, Portugal

e-mail: pandrade@ff.up.pt

Tel.: +351-222078935

Fax: +351-222003977

V. Vrchovská

Department of Pharmacognosy, Faculty of Pharmacy, Charles

University,

Heyrovského 1203 ,

50005 Hradec Králové, Czech Republic

J. A. Pereira

CIMO/ESAB, Quinta de Sta Apolónia,

Apartado 1172,

5301-855 Bragança, Portugal capacity to act as a scavenger of $\mathrm{DPPH}^{\bullet}$ radical and reactive oxygen species (superoxide radical, hydroxyl radical and hypochlorous acid), exhibiting antioxidant capacity in a concentration dependent manner against all radicals.

Keywords Tronchuda cabbage internal leaves - Brassica oleracea L. var. costata DC · Phenolics · Organic acids · Antioxidant capacity

\section{Inoduction}

An increasing amount of evidence shows that the consumption of fruits and vegetables is, in general, beneficial to health due to the protection provided by the antioxidant compounds contained in them $[1,2]$. In fact, the presence of phytochemicals, in addition to vitamins and provitamins, has been considered of great nutritional interest in the prevention of chronic diseases, such as cancer, arteriosclerosis, nephritis, diabetes mellitus, rheumatism, ischemic and cardiovascular diseases and also in the aging process, in which oxidants or free radicals are involved [3-6]. Among the natural antioxidant molecules, it can be found the liposoluble vitamins $\mathrm{A}$ and $\mathrm{E}, \beta$-carotene, the water-soluble vitamin $\mathrm{C}$, and a wide range of molecules generally termed phenolic compounds, including phenolic acids, flavonoids, glucosides and esters [7-9]. Synergistically or additively, these dietary antioxidants provide bioactive mechanisms to reduce free radical-induced oxidative stress [3]. Although the organism possesses such defence mechanisms as enzymes and antioxidant molecules [10,11], oxidative stress results either from a decrease of natural cell antioxidant capacity or from an increased amount of reactive oxygen species (ROS). When the balance between oxidants and antioxidants is broken by the overproduction of free radicals beyond the organism control, it can cause irreversible oxidative damage [12].

Nowadays consumers are aware of the need for a constant supply of phytochemical-containing plants to get the most complete antioxidant support for diseases prevention. Really, through overlapping or complementary 
effects, the complex mixture of the compounds present in fruits and vegetables provides a more effective protective action on health than single phytochemicals $[3,13]$. This is ascribed to the fact that different plants have distinct compounds contents, with several structures, thus offering different protective mechanisms at different levels. So, to obtain optimal health benefits, it has been suggested a diet composed of a variety of phytochemical sources, such as fruits and vegetables $[3,8,13]$.

Brassica vegetables, including all cabbage-like ones, are consumed in enormous quantities throughout the world and are important in human nutrition. They are reported to reduce the risks of some cancers especially due to its content of glucosinolates and their derived products [14-17], although phenolic compounds are also considered to contribute to this capacity [18-20]. Some Brassica oleracea varieties, namely cauliflower $[21,22]$, broccoli $[8,9,22$, $23]$ and several cabbages [2, 3, 13, 22, 24] have already been studied for their antioxidant capacity in different experimental models.

Tronchuda cabbage (Brassica oleracea L. var. costata DC) plant resembles a thick-stemmed collard with large floppy leaves. Leaves are close together, round, smooth and slightly notched at the margins. Regarding the organoleptic properties, internal and external leaves are considerably different: internal leaves are pale yellow and are tender and sweeter than the dark green external leaves, which may influence the consumer's choice. Due to these characteristics internal leaves are eaten raw in salads or, most usually, cooked. As far as we know, only the phenolic composition of the external leaves has been described, consisting of complex flavonol glycosides [25], and nothing has been reported about the antioxidant capacity of tronchuda cabbage.

The objectives of this study were to define the phenolics and organic acids composition and to evaluate the antioxidant potential of tronchuda cabbage internal leaves hot-water extract, since this is the way how it is most consumed. Phenolic profile was established by reversed-phase HPLC-DAD-ESI-MS ${ }^{n}$ and HPLC-DAD analysis. Organic acids were determined by HPLC/UV. The antioxidant activity was assessed by the capacity to act as scavenger of DPPH radical and reactive oxygen species (superoxide radical, hydroxyl radical and hypochlorous acid). A comparison with tronchuda cabbage external leaves was also undertaken.

\section{Materials and methods}

Standards and reagents

The standards were from Sigma (St. Louis, MO) and from Extrasynthése (Genay, France). Methanol, ammonium acetate and acetic acid were obtained from Merck (Darmstadt, Germany) and sulphuric acid from Pronalab (Lisboa, Portugal). The water was treated in a Milli-Q water purification system (Millipore, Bedford, MA). DPPH, xanthine, xanthine oxidase (XO) grade I from buttermilk
(EC 1.1.3.22), $\beta$-nicotinamide adenine dinucleotide $(\mathrm{NADH})$, phenazine methosulfate (PMS), nitroblue tetrazolium chloride (NBT), ferric chloride anhydrous $\left(\mathrm{FeCl}_{3}\right)$, ethylenediaminetetraacetic acid disodium salt (EDTA), ascorbic acid, trichloroacetic acid, thiobarbituric acid, deoxyribose, sodium hypochlorite solution with $4 \%$ available chlorine $(\mathrm{NaOCl}), 5,5^{\prime}$-dithiobis(2-nitrobenzoic acid) (DTNB) were obtained from Sigma (St. Louis, MO).

\section{Plant material and sampling}

Tronchuda cabbages were grown under organic practices, certified by the national authority (Instituto de Desenvolvimento Rural e Hidráulica), following the guidelines of Council Regulation (EEC) no 2092/91 of 24 June 1991 (organic production). Only organic fertilization was made with sheep manure and no phytosanitary treatments were applied. After harvesting in October 2004, three plants randomly selected were immediately transported to the laboratory where external and internal leaves were separated. The internal leaves were freezed and lyophilised (Modulyo 4 K Freeze Dryer Ed wards) and the three lyophilised materials were powdered, mixed and kept in an exsicator, in the dark. The same procedure was applied to the external leaves.

\section{Sample preparation}

The identification of the phenolic compounds in tronchuda cabbage internal leaves was performed using a hydromethanolic extract: the lyophilised plant material (ca. $0.5 \mathrm{~g}$ ) was thoroughly mixed with $5 \mathrm{ml}$ methanol-water (1:1), ultra-sonicated, centrifuged and filtered.

For antioxidant activity assays both internal and external leaves extracts were prepared by putting $3.0 \mathrm{~g}$ of lyophilised plant material in $600 \mathrm{ml}$ of boiling water. The mixture was boiled for an hour and then filtered over a Büchner funnel. The resulting extracts were then lyophilised and a yield of 1.6 and $1.2 \mathrm{~g}$ were obtained for internal and external leaves, respectively. The lyophilised extracts were kept in an exsicator, in the dark. For organic acids analysis the lyophilised extracts were redisssolved in sulphuric acid $0.01 \mathrm{~N}$. For phenolic compounds determination water was used for the redissolution of the lyophilised extracts.

\section{HPLC analysis of organic acids}

The separation was carried out as previously reported [26] with some modifications. The system consisted of an analytical HPLC unit (Gilson) with an ion exclusion column, Nucleogel Ion 300 OA $(300 \mathrm{~mm} \times 7.7 \mathrm{~mm})$ in conjunction with a column-heating device set at $30^{\circ} \mathrm{C}$. Elution was carried out isocratically with sulphuric acid $0.01 \mathrm{~N}$ as mobile phase with a flow rate of $0.2 \mathrm{ml} \mathrm{min}^{-1}$. The injection volume was $20 \mu \mathrm{l}$. Detection was performed with an UV detector set at $214 \mathrm{~nm}$. Organic acids quantification was 
achieved by the absorbance recorded in the chromatograms relative to external standards.

HPLC-DAD-ESI-MS ${ }^{n}$ qualitative analysis of phenolics in internal leaves

Chromatographic separations were carried out on a $250 \mathrm{~mm} \times 4 \mathrm{~mm}, 5-\mu \mathrm{m}$ particle size, RP-18 LiChroCART (Merck, Darmstadt, Germany) column protected with a $4 \mathrm{~mm} \times 4 \mathrm{~mm}$ LiChroCART guard column using acetic acid $1 \%$ (A) and methanol (B) as solvents, starting with $20 \%$ B and using a gradient to obtain $50 \% \mathrm{~B}$ at $30 \mathrm{~min}$ and $80 \% \mathrm{~B}$

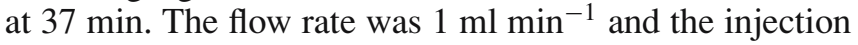
volumes varied between 10 and $50 \mu 1$.

The HPLC system was equipped with an Agilent 1100 Series diode array and a mass detector in series (Agilent Technologies, Waldbronn, Germany). It consisted of a G1312A binary pump, an G1313A autosampler, a G1322A degasser and a G1315B photodiode array detector con- trolled by a ChemStation software (Agilent, v. 08.03). Spectroscopic data from all peaks were accumulated in the range 240-400 nm, and chromatograms were recorded at $330 \mathrm{~nm}$. The mass detector was a G2445A Ion-Trap Mass Spectrometer equipped with an electrospray ionisation (ESI) system and controlled by LCMSD software (Agilent, v. 4.1.). Nitrogen was used as nebulizing gas at a pressure of $65 \mathrm{psi}$ and the flow was adjusted to $111 \mathrm{~min}^{-1}$. The heated capillary and voltage were maintained at $350^{\circ} \mathrm{C}$ and $4 \mathrm{kV}$, respectively. The full-scan mass covered the range from $\mathrm{m} / \mathrm{z} 90$ up to $\mathrm{m} / \mathrm{z}$ 2000. Collision-induced fragmentation experiments were performed in the ion trap using helium as collision gas, with voltage ramping cycles from 0.3 up to $2 \mathrm{~V}$. MS data were acquired in the negative ionisation mode. $\mathrm{MS}^{n}$ data were achieved in the automatic mode on the more abundant fragment ion in $\mathrm{MS}^{n-1}$. Tables 1-3 show the most frequent ions which characterise the fragmentation of the compounds. Other ions were found but they have not been included due to their low significance on the MS behaviour ions. The classical nomenclature for glycoconjugates was

Table 1 Rt, UV, -MS: $[\mathrm{M}-\mathrm{H}]^{-},-\mathrm{MS}^{2}[\mathrm{M}-\mathrm{H}]^{-}$and $-\mathrm{MS}^{3}\left[(\mathrm{M}-\mathrm{H}) \rightarrow \mathrm{Y}_{0}^{7}(-162)\right]^{-}$data of flavonoid-3-O-Soph-7-O-Glc and flavonoid-3-OSoph

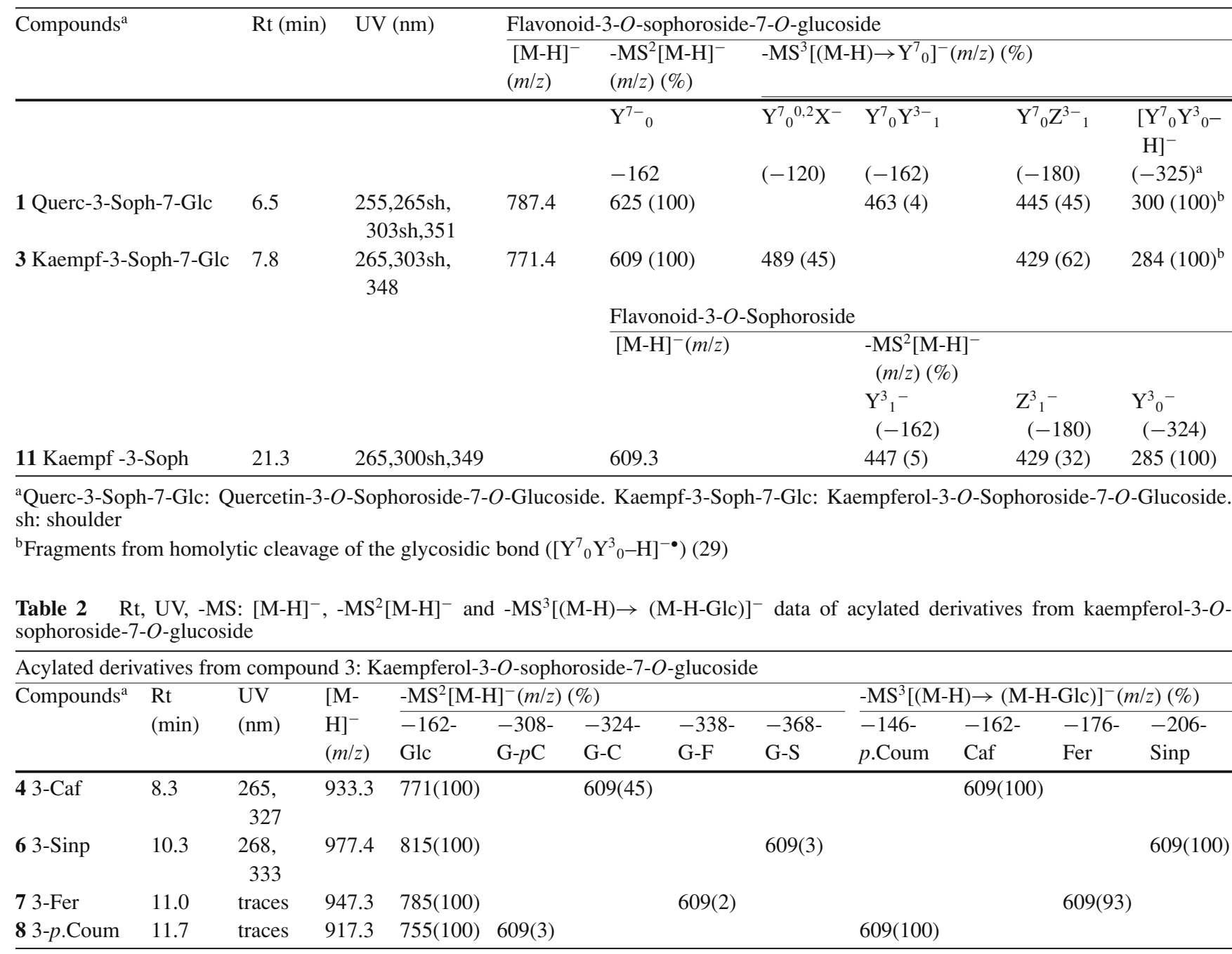

${ }^{\mathrm{a}} \mathrm{G}$ (Glc): Glucosyl. pC (p.Coum): pCoumaroyl. C (Caf): Caffeoyl. F (Fer): Feruloyl. S (Sinp): Sinapoyl 


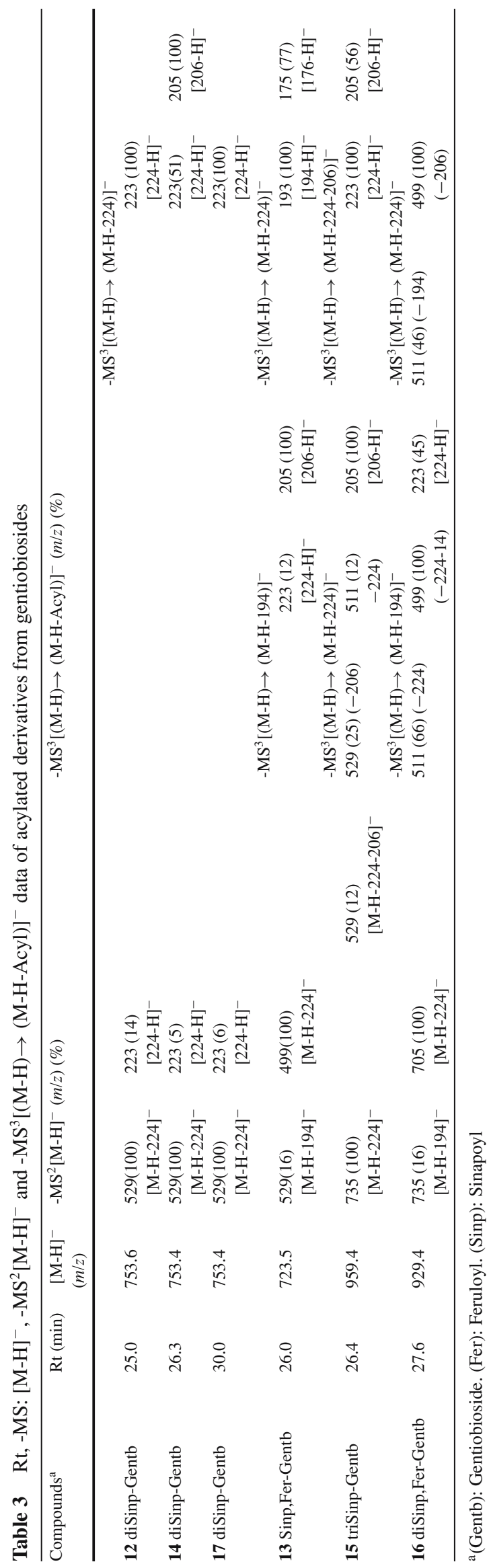

adopted to designate the fragment ions. The ions ${ }^{k, l} \mathrm{X}_{j}, \mathrm{Y}^{n}{ }_{j}$, $\mathrm{Z}^{n}{ }_{j}$ represent fragments still containing the flavonoid aglycone, where $j$ is the number of the interglycosidic bond broken, counted from the aglycon, $n$ represents the position of the phenolic hydroxyl where the oligosaccharide is attached, and the $k$ and $l$ denote the cleavage within the carbohydrate rings. The ions obtained through the fragmentation of flavonoids with glycosylation in two different phenolic hydroxyls were labelled with a superscript number that indicates the position of these hydroxyls [27-29].

In order to enhance ionisation, mainly for the compounds 2 and $\mathbf{5}$, ammonium acetate $10 \mathrm{mM}$ with a flow of $2 \mathrm{ml} \mathrm{h}^{-1}$ was added to the eluent with a $\mathrm{T}$ join between the UV and the mass detector.

\section{HPLC-DAD quantitative analysis of phenolics}

Twenty microliters of the internal leaves hot-water extract were analysed using a HPLC unit (Gilson) and a RP18 LiChroCART (Merck, Darmstadt, Germany) column ( $250 \mathrm{~mm} \times 4 \mathrm{~mm}, 5-\mu \mathrm{m}$ particle size). The solvent system was the same as described for the qualitative analysis.

The quantification of the phenolic compounds present in external leaves hot-water extract was performed according to a described procedure [25], using a HPLC unit (Gilson) and a $250 \mathrm{~mm} \times 4.6 \mathrm{~mm}$ i.d., $5 \mu \mathrm{m}$ Spherisorb ODS2 column (Waters, Milford). The solvent system was a mixture of formic acid 5\% in water (A) and methanol (B), with a flow rate of milliliters per minute, and the gradient was as follows: 0 min: $10 \% \mathrm{~B} ; 25 \mathrm{~min}: 20 \% \mathrm{~B}$; $40 \mathrm{~min}: 50 \% \mathrm{~B}$; 45 min: 50\% B; 46 min: $90 \%$ B; 50 min: $90 \%$ B; 55 min: 100\% B; 58 min: $100 \%$ B; and 60 min: 10\% B.

In both cases detection was achieved with a Gilson diode array detector. Spectral data from all peaks were accumulated in the range of 200-400 nm, and chromatograms were recorded at $330 \mathrm{~nm}$. The data were processed on Unipoint system Software (Gilson Medical Electronics, Villiers le Bel, France). Peak purity was checked by the software contrast facilities.

Phenolic compounds quantification was achieved by the absorbance recorded in the chromatograms relative to external standards. Since standards of the compounds identified in the internal leaves hot-water extract were not commercially available quercetin 3-O-sophoroside-7-O-glucoside was quantified as rutin, 3- and 4- $p$-coumaroylquinic acids were quantified as $p$-coumaric acid, the kaempferol derivatives were quantified as kaempferol 3-O-rutinoside and sinapic acid derivatives as sinapic acid.

\section{DPPH scavenging activity}

The antiradical activity of the extracts was determined spectrophotometrically in an ELX808 IU Ultra Microplate Reader (Bio-Tek Instruments, Inc), by monitoring the disappearance of DPPH at $515 \mathrm{~nm}$, according to a described procedure [30]. For each extract, a dilution series (five different concentrations) was prepared in a 96-well plate. 
The reaction mixtures in the sample wells consisted of $25 \mu \mathrm{l}$ hot-water extract and $200 \mu \mathrm{l}$ DPPH radical dissolved in methanol. The plate was incubated for $30 \mathrm{~min}$ at room temperature. Three experiments were performed in triplicate.

Evaluation of superoxide radical scavenging activity

Antiradical activity was determined spectrophotometrically in an ELX808 IU Ultra Microplate Reader (BioTek Instruments, Inc), by monitoring the effect of the lyophilised extracts on the $\mathrm{O}_{2}{ }^{\bullet-}$-induced reduction of NBT at $562 \mathrm{~nm}$. The direct reduction of NBT by the extracts was checked without the superoxide radical generation systems and no effect was observed.

\section{Non-enzymatic assay}

Superoxide radicals were generated by the NADH/PMS system according to a described procedure [31]. All components were dissolved in phosphate buffer $19 \mathrm{mM}, \mathrm{pH}$ 7.4. Three experiments were performed in triplicate.

\section{Enzymatic assay}

Superoxide radicals were generated by the xanthine/xanthine oxidase (X/XO) system following a described procedure [31]. Xanthine was dissolved in $\mathrm{NaOH}$ $1 \mu \mathrm{M}$ and subsequently in phosphate buffer $50 \mathrm{mM}$ with EDTA $0.1 \mathrm{mM}, \mathrm{pH} 7.8$, xanthine oxidase in EDTA $0.1 \mathrm{mM}$ and the other components in phosphate buffer $50 \mathrm{mM}$ with EDTA $0.1 \mathrm{mM}, \mathrm{pH}$ 7.8. Three experiments were performed in triplicate.

\section{Effect on xanthine oxidase activity}

The effect of the lyophilised extracts on xanthine oxidase activity was evaluated by measuring the formation of uric acid from xanthine in a double beam spectrophotometer (He $\lambda$ ios $\alpha$, Unicam), at room temperature. The reaction mixtures contained the same proportion of components as in the enzymatic assay for superoxide radical scavenging activity, except NBT, in a final volume of $750 \mu$ l. The absorbance was measured at $295 \mathrm{~nm}$ for $2 \mathrm{~min}$. Three experiments were performed in triplicate.

\section{Hydroxyl radical assay}

The deoxyribose method for determining the scavenging effect of the hot-water extracts on hydroxyl radicals was performed according to a described procedure [32] in a double beam spectrophotometer (He $\lambda$ ios $\alpha$, Unicam). Reaction mixtures contained $50 \mu \mathrm{M}$ ascorbic acid, $40 \mu \mathrm{M}$ $\mathrm{FeCl}_{3}, 2 \mathrm{mM}$ EDTA, $2.8 \mathrm{mM} \mathrm{H} \mathrm{O}_{2}, 2.8 \mathrm{mM}$ deoxyribose and lyophilised extracts. All components were dissolved in $\mathrm{KH}_{2} \mathrm{PO}_{4}-\mathrm{KOH}$ buffer $10 \mathrm{mM}, \mathrm{pH}$ 7.4. This assay was also performed either without ascorbic acid or EDTA, in order to evaluate the extracts pro-oxidant and metal chelation potential, respectively. Three experiments were performed in triplicate.

\section{Hypochlorous acid scavenging activity}

The inhibition of hypochlorous acid-induced 5-thio-2nitrobenzoic acid (TNB) oxidation to 5,5'-dithiobis(2nitrobenzoic acid) was performed according to a described procedure [32], in a double beam spectrophotometer (He $\lambda$ ios $\alpha$, Unicam). Hypochlorous acid and TNB were prepared immediately before use. Scavenging of hypochlorous acid was ascertained by using lipoic acid as a reference scavenger, which scavenged $\mathrm{HOCl}$ in a concentration dependent manner (data not shown). Three experiments were performed in triplicate.

\section{Results and discussion}

Characterization of the internal leaves phenolic compounds

The screening by HPLC-DAD-ESI-MS ${ }^{n}$ of the hydromethanolic extract of tronchuda cabbage internal leaves (Fig. 1A) revealed the presence of several hydroxycinnamic acid derivatives, with the exception of compounds 1, 3 and 11, which UV and MS spectra (Table 1) coincided with those of quercetin 3-O-sophoroside-7$O$-glucoside, kaempferol 3-O-sophoroside-7- $O$-glucoside and kaempferol 3-O-sophoroside, respectively. These compounds had already been characterized in other Brassica vegetables, namely cauliflower [33] and broccoli [34], and their MS fragmentation allows them to be differentiated from other possible isomers [35]. Compounds 4, 6, 7 and $\mathbf{8}$ are monoacylated derivatives of compound $\mathbf{3}$, being caffeic, sinapic, ferulic and $p$-coumaric acids linked to the sophorose, respectively (Table 2). These compounds had also been reported in the previously mentioned Brassica species and exhibit a characteristic MS fragmentation pathway [34].

The HPLC-DAD-ESI-MS ${ }^{n}$ analysis showed an abundance of non-flavonoidic hydroxycinnamic acid derivatives. Thus, in the first part of the chromatogram (Fig. 1A) compound $\mathbf{2}$ is the main one, which fragmentation (-MS: $337[\mathrm{M}-\mathrm{H}]^{-},-\mathrm{MS}^{2}[\mathrm{M}-\mathrm{H}]^{-}: 163(100 \%)$ ) is coincident with that of 3-p-coumaroylquinic acid [36]. Sinapoyl glucoside acid (5) (-MS: $385[\mathrm{M}-\mathrm{H}]^{-}, \mathrm{MS}^{2}$ : 247 (51\%), 223 ([M-

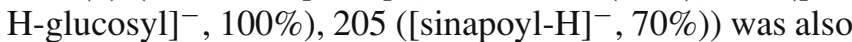
detected.

In the second part of the chromatogram (Fig. 1A) some compounds corresponding to sinapic and ferulic acids esterified with a dihexose were noticed (compounds 12-17). Several conjugates of gentiobiose (glucosil $1 \rightarrow 6$ glucose) 

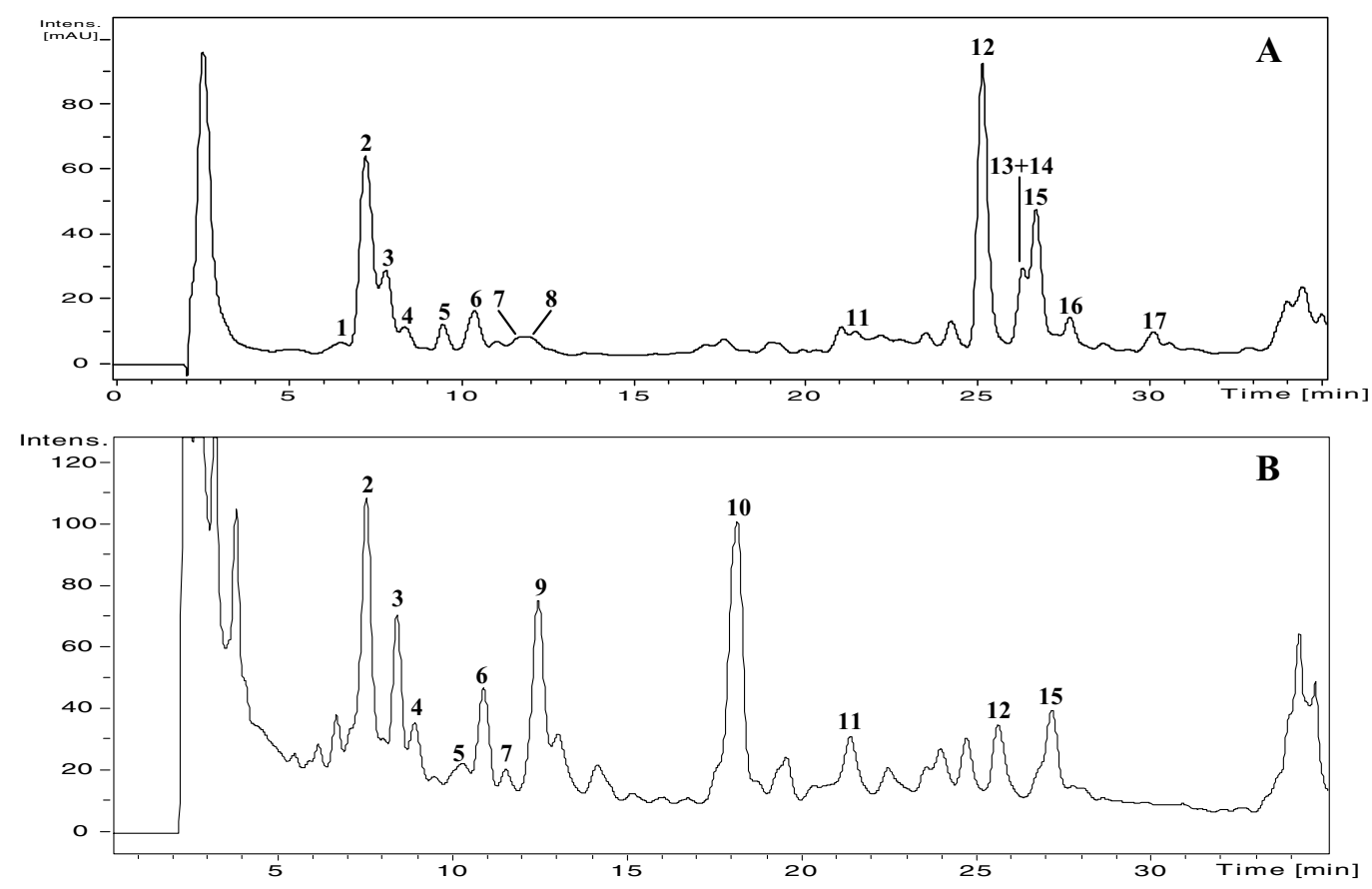

Fig. 1 HPLC-DAD phenolic profile of tronchuda cabbage internal leaves (A) hydro-methanolic extract and (B) hotwater extract. Detection at $330 \mathrm{~nm}$. Peaks: (1) quercetin 3$O$-sophoroside-7- $O$-glucoside; (2) 3-p-coumaroylquinic acid; (3) kaempferol 3-O-sophoroside-7-O-glucoside; (4) kaempferol 3$O$-(caffeoyl)-sophoroside-7- $O$-glucoside; (5) sinapoyl glucoside acid; (6) kaempferol 3-O-(sinapoyl)-sophoroside-7- $O$-glucoside;
(7) kaempferol 3-O-(feruloyl)-sophoroside-7-O-glucoside; (8) kaempferol 3-O-( $p$-coumaroyl)-sophoroside-7-O-glucoside; (9) 4p-coumaroylquinic acid; (10) sinapic acid; (11) kaempferol 3$O$-sophoroside; (12) 1,2-disinapoylgentiobiose; (13) 1-sinapoyl2-feruloylgentiobiose; (14) isomer of 1,2-disinapoylgentiobiose; (15) 1,2,2'-trisinapoylgentiobiose; (16) 1,2'-disinapoyl-2-feruloylgentiobiose; (17) isomer of 1,2-disinapoylgentiobiose

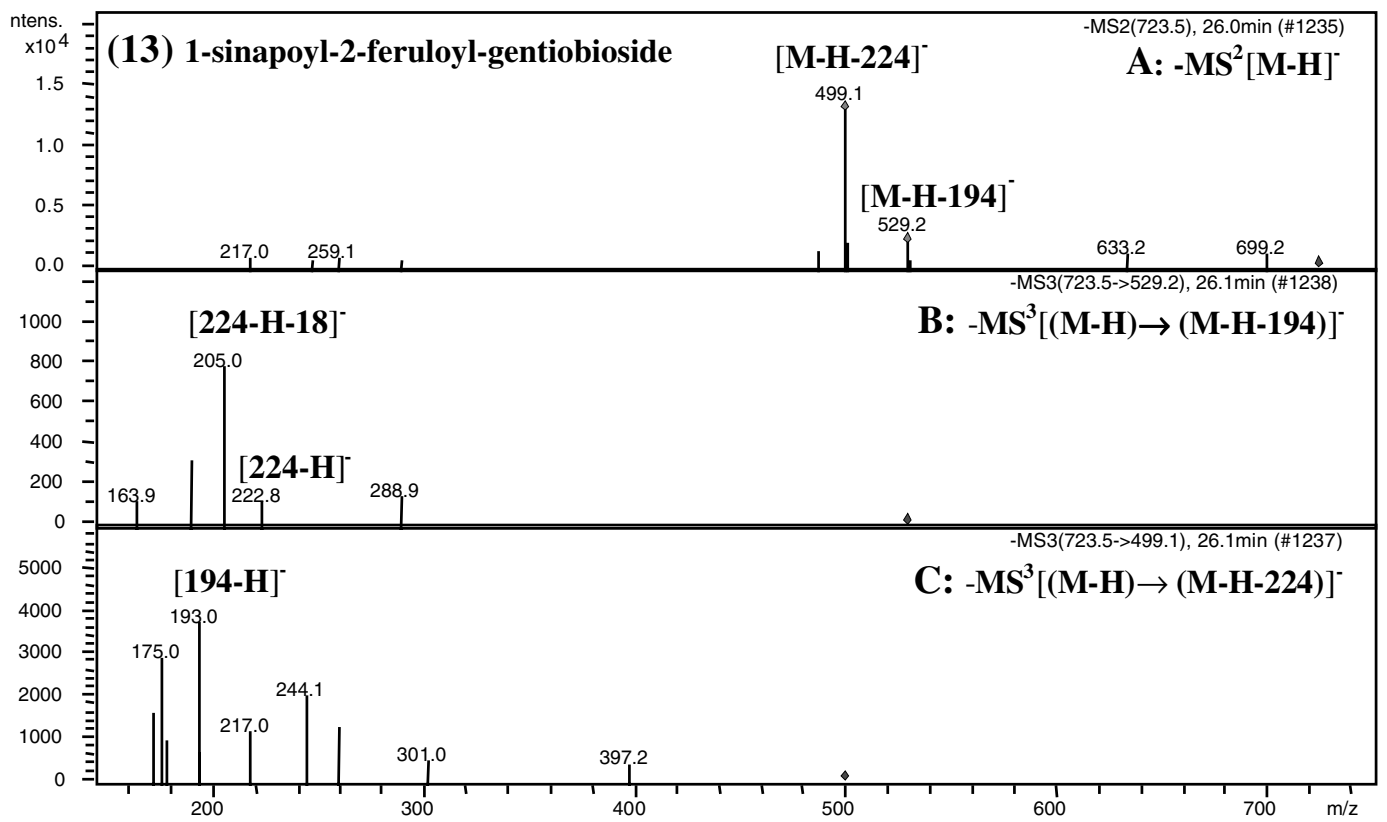

Fig. 2 Negative $\mathrm{MS}^{n}$ analysis of 1-sinapoyl-2-feruloylgentiobiose (13). (A) $\mathrm{MS}^{2}[\mathrm{M}-\mathrm{H}]^{-}$; (B) $\mathrm{MS}^{3}[(\mathrm{M}-\mathrm{H}) \rightarrow(\mathrm{M}-\mathrm{H}-\mathrm{Fer})]^{-}$; (C) $\mathrm{MS}^{3}[(\mathrm{M}-$ $\mathrm{H}) \rightarrow(\mathrm{M}-\mathrm{H}-$ Fer-Sinp $)]^{-}$

and hydroxycinnamic acids had been characterized before in broccoli [37] and, therefore, the compounds detected in this work may be coincident with them. In addition, this type of compounds is very common in Brassica species $[33,34,38]$. From the study of the distinct $\mathrm{MS}^{n}$ frag- mentation pathways (Table 3) it could be observed, in all cases, the loss of 224 amu from the deprotonated molecular ion, corresponding to sinapic acid. Those compounds that besides sinapic acid presented ferulic acid (compounds $\mathbf{1 3}$ and 16) also displayed the loss of this acid (194 amu) 


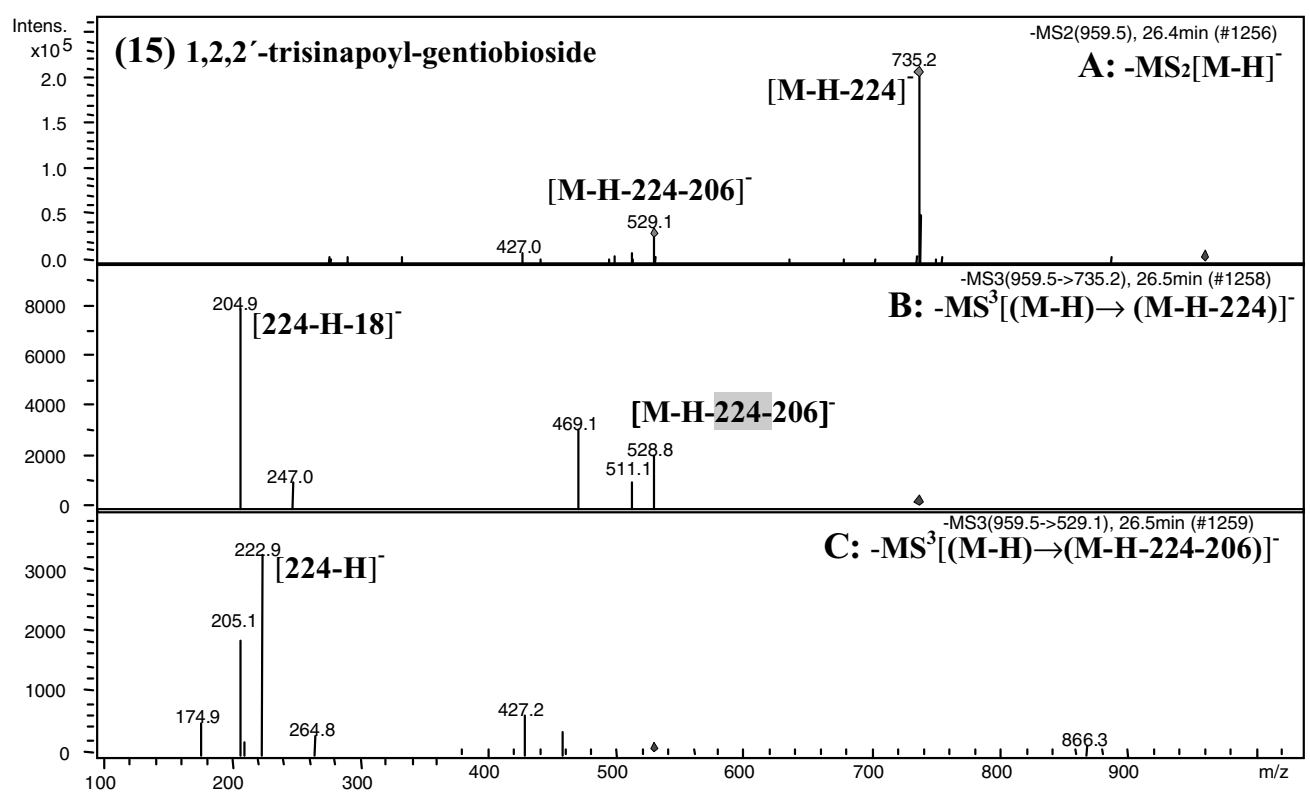

Fig. 3 Negative $\mathrm{MS}^{n}$ analysis of 1,2,2'-trisinapoylgentiobiose (15). (A) $\mathrm{MS}^{2}[\mathrm{M}-\mathrm{H}]^{-}$; (B) $\mathrm{MS}^{3}\left[\left(\mathrm{M}-\mathrm{H} \rightarrow(\mathrm{M}-\mathrm{H}-\operatorname{sinp}]^{-}\right.\right.$; $(\mathbf{C}) \mathrm{MS}^{3}[(\mathrm{M}-\mathrm{H}) \rightarrow$ (M-H-Sinp-Sinp) $]^{-}$

(Fig. 2A, Table 3). In $\mathrm{MS}^{3}[(\mathrm{M}-\mathrm{H}) \rightarrow(\mathrm{M}-\mathrm{H}-\mathrm{Acyl})]^{-}$ (Table 3 ) it could be observed, besides an additional loss of acyls, the ions corresponding to the acids: $\mathrm{m} / \mathrm{z} 223$ [sinapic acid-H] ${ }^{-}, 205$ [sinapic acid-H-18] ${ }^{-}, 193$ [ferulic acid-H] ${ }^{-}$, 175 [ferulic acid-H-18] $^{-}$(Figs. 2B, C, 3B and C). Other ions resulting from the partial fragmentation of the sugars were observed but not included in Table 3. Tentatively, we can consider that the detected compounds are coincident with those previously described [37] and were identified as 1,2-disinapoylgentiobiose (12), 1-sinapoyl2-feruloylgentiobiose (13), 1,2,2'-trisinapoylgentiobiose (15), 1,2'-disinapoyl-2-feruloylgentiobiose (16). Compounds $\mathbf{1 4}$ and $\mathbf{1 7}$ are 1,2-disinapoylgentiobiose isomers.
In the hydroalchoolic extract synapic acid (10) was also detected in trace amounts (Fig. 1A).

The aqueous lyophilised extract of tronchuda cabbage internal leaves presented a similar composition. However, in this extract it were not found quercetin 3-O-sophoroside-7-O-glucoside (1), kaempferol 3-O-(pcoumaroyl)-sophoroside-7- $O$-glucoside (8), 1-sinapoyl2-feruloylgentiobiose (13) and the isomers of 1,2disinapoylgentiobiose (14 and 17). Besides, in this aquous extract 4- $p$-coumaroylquinic acid (9) was present (Fig. 1B).

All these compounds are identified in tronchuda cabbage for the first time, with the exceptions of kaempferol
Table 4 Phenolic composition of tronchuda cabbage internal leaves hot-water extract $(\mathrm{mg}$ of phenolic compound $\mathrm{kg}^{-1}$ of lyophilised extract) ${ }^{\mathrm{a}}$

\begin{tabular}{llll}
\hline & Phenolic compound & Mean & SD \\
\hline $\mathbf{1}$ & Quercetin 3- $O$-sophoroside-7- $O$-glucoside & nd & \\
$\mathbf{2}$ & 3-p-Coumaroylquinic acid & 189.0 & 1.4 \\
$\mathbf{3}$ & Kaempferol 3- $O$-sophoroside-7- $O$-glucoside & 287.8 & 8.8 \\
$\mathbf{4}$ & Kaempferol 3-(cafeoyl)-sophoroside-7- $O$-glucoside & 120.5 & 3.7 \\
$\mathbf{5}$ & Sinapoyl glucoside acid & 25.7 & 0.2 \\
$\mathbf{6}$ & Kaempferol 3-(sinapoyl)-sophoroside-7- $O$-glucoside & 180.7 & 5.5 \\
$\mathbf{7}$ & Kaempferol 3-(feruloyl)-sophoroside-7- $O$-glucoside & 53.6 & 1.6 \\
$\mathbf{8}$ & Kaempferol 3-(p-coumaroyl)-sophoroside-7- $O$-glucoside & nd & \\
$\mathbf{9}$ & 4- $p$-Coumaroylquinic acid & 126.0 & 0.9 \\
$\mathbf{1 0}$ & Sinapic acid & 180.1 & 1.1 \\
$\mathbf{1 1}$ & Kaempferol 3- $O$-sophoroside & 100.4 & 3.1 \\
$\mathbf{1 2}$ & 1,2-Disinapoylgentiobioside & 51.5 & 0.3 \\
$\mathbf{1 3}$ & 1-Sinapoyl-2-feruloylgentiobiose & nd & \\
$\mathbf{1 4}$ & Isomer of 1,2-disinapoylgentiobioside & nd & \\
$\mathbf{1 5}$ & 1,2,2'-trisinapoylgentiobiose & 62.9 & 0.4 \\
$\mathbf{1 6}$ & 1-2'-disinapoyl-2-feruloylgentiobiose & 11.4 & 0.1 \\
$\mathbf{1 7}$ & Isomer of 1,2-disinapoylgentiobioside & nd & $\Sigma 1389.6$ \\
\end{tabular}

${ }^{a}$ Results are expressed as mean of three determinations. SD standard deviation, $\sum$, sum of the determined phenolic compounds 

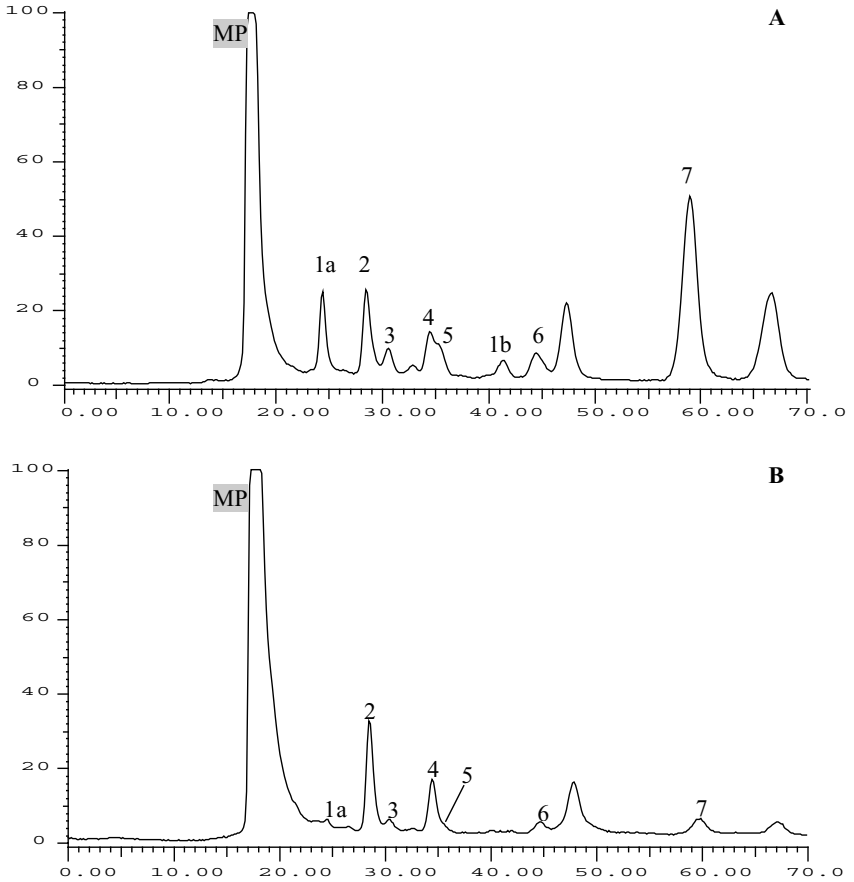

Fig. 4 HPLC-UV organic acid profile of tronchuda cabbage (A) internal and (B) external leaves hot-water extracts. Detection at $214 \mathrm{~nm}$. Peaks: (MP) mobile phase; (1a and $\mathbf{1 b}$ ) aconitic acid isomers; (2) citric acid; (3) ascorbic acid; (4) malic acid; (5) quinic acid; (6) shikimic acid; (7) fumaric acid

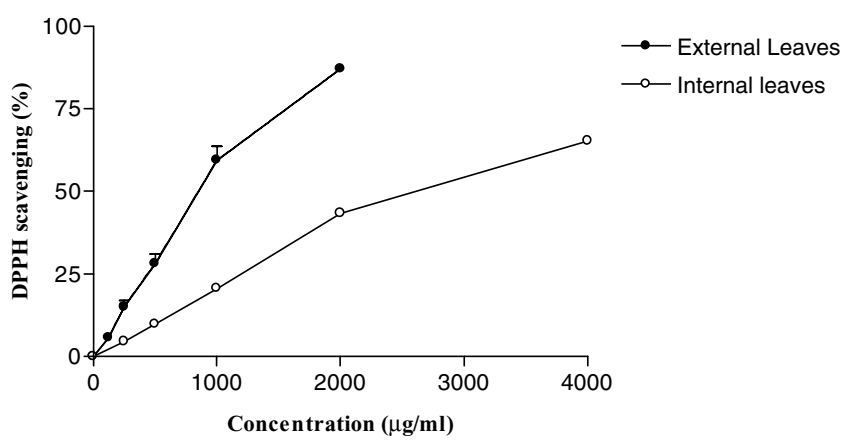

Fig. 5 Effect of tronchuda cabbage internal and external leaves on $\mathrm{DPPH}$ reduction. Values show mean $\pm \mathrm{SE}$ from three experiments performed in triplicate

3- $O$-sophoroside-7- $O$-glucoside (3) and kaempferol 3-Osophoroside (11), previously described in its external leaves [25].

Internal leaves phenolic compounds quantitative analysis

In order to a get a better characterization of the aqueous lyophilised extract of tronchuda cabbage internal leaves used in antioxidant assays, its phenolic compounds were quantified by HPLC/DAD. The results showed a high content of phenolics (ca. $1.4 \mathrm{~g} \mathrm{~kg}^{-1}$, dry basis) (Table 4), being kaempferol 3-O-sophoroside-7- $O$-glucoside $(3)$ the main compound (ca. $21 \%$ of total identified compounds),
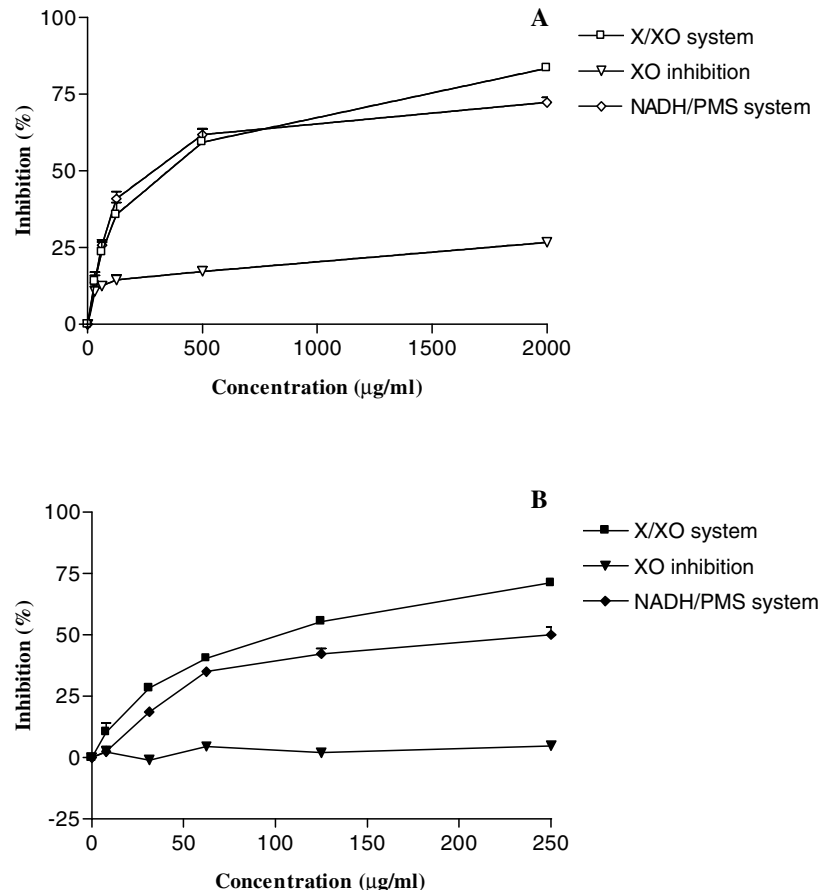

Fig. 6 Effect of tronchuda cabbage (A) internal and (B) external leaves against superoxide radical generated in an enzymatic and nonenzymatic systems and on XO activity. Values show mean \pm SE from three experiments performed in triplicate

followed by 3-p-coumaroylquinic acid (2) (ca. 14\% of total phenolics). 1,2'-Disinapoyl-2-feruloylgentiobiose (16) was present in the lowest amount (ca. 1\% of total identified compounds).

Sinapic acid (10), which was detected only in vestigial amounts in the hydro-methanolic extract, represented $13 \%$ of the identified phenolics in the aqueous lyophilised extract. The presence of higher amounts of sinapic acid and the existence of 4-p-coumaroyl quinic acid (9) in the hotwater extract may be attributed to a higher solubility of these compounds in the boiling water. Decomposition of the sinapic acid derivatives can partially explain the differences observed in the chromatograms of both methanolic and hot-water extracts.

Identification and quantification of organic acids by HPLC/UV

Tronchuda cabbage internal leaves presented a chemical profile composed by seven identified organic acids: aconitic, citric, ascorbic, malic, quinic, shikimic and fumaric acids (Fig. 4A). All these compounds are described for the first time in this cabbage. None of the samples presented oxalic, ketoglutaric, succinic, lactic, acetic, pyruvic, malonic or tartaric acids. The lyophilised extract exhibited a high content of organic acids (ca. $23 \mathrm{~g} \mathrm{~kg}^{-1}$ ), in which citric acid was the main compound, representing ca. $43 \%$ of total identified organic acids, followed by the pair malic plus quinic acids (ca. $28 \%$ of total acids) 
Table 5 Organic acids in tronchuda cabbage leaves hot-water extracts ( $\mathrm{mg}$ of organic acid $\mathrm{kg}^{-1}$ of lyophilised extract) ${ }^{\mathrm{a}}$

${ }^{\text {a }}$ Results are expressed as means
of three determinations; SD
standard deviation, $\sum$, sum of
the determined organic acids
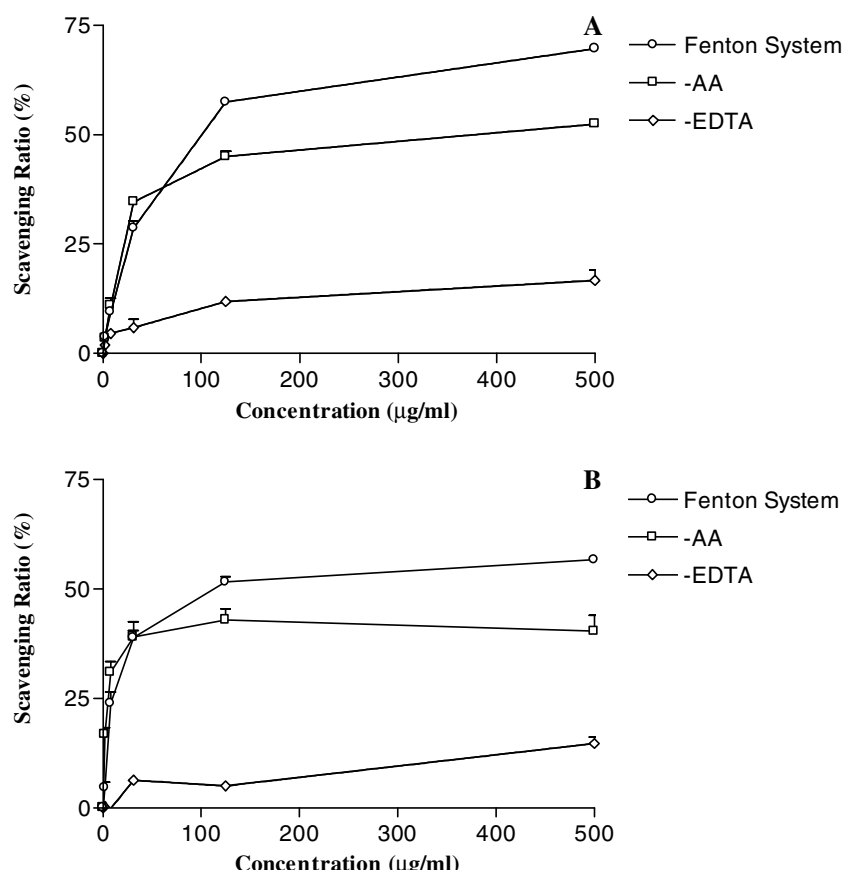

Fig. 7 Tronchuda cabbage (A) internal and (B) external leaves nonspecific hydroxyl radical scavenging activity, pro-oxidant activity (-AA) and specific hydroxyl radical scavenging (-EDTA). Values show mean $\pm \mathrm{SE}$ from three experiments performed in triplicate

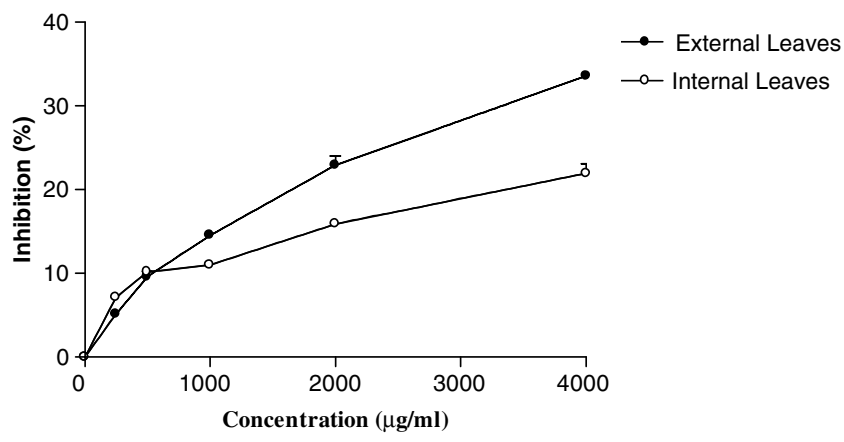

Fig. 8 Effect of tronchuda cabbage internal and external leaves on the oxidation of TNB by $\mathrm{HOCl}$. Values show mean \pm SE from three experiments performed in triplicate

(Table 5). Shikimic acid was the compound present in minor amounts, accounting for ca. $0.2 \%$ of compounds (Table 5).

\section{Antioxidant activity}

The $\mathrm{DPPH}^{\bullet}$ assay constitutes a screening method currently used to provide basic information on the antiradical activity of extracts. Reduction of DPPH ${ }^{\bullet}$ by antioxidants leads to a loss of absorbance at $515 \mathrm{~nm}$ [39]. In the present work the lyophilised extract of tronchuda cabbage internal leaves

Table 6 Phenolic composition of tronchuda cabbage external leaves hot-water extract (mg of phenolic compound $\mathrm{kg}^{-1}$ of lyophilised extract) ${ }^{\mathrm{a}}$

\begin{tabular}{|c|c|c|c|}
\hline & Phenolic compound & Mean & SD \\
\hline 2 & 3-p-Coumaroylquinic acid & 481.8 & 8.6 \\
\hline 3 & Kaempferol 3-O-sophoroside-7- $O$-glucoside & 852.2 & 14.0 \\
\hline $\begin{array}{c}18+ \\
19\end{array}$ & $\begin{array}{l}\text { Kaempferol 3- } O \text {-sophorotrioside-7- } O \text {-glucoside }+ \text { kaempferol } \\
\text { 3- } O \text {-(methoxycaffeoyl/caffeoyl)-sophoroside-7- } O \text {-glucoside }\end{array}$ & 452.6 & 12.3 \\
\hline 20 & Kaempferol 3-O-sophorotrioside-7- $O$-sophoroside & 445.3 & 6.1 \\
\hline 23 & Kaempferol 3-O- (feruloyl/caffeoyl)-sophoroside-7-O-glucoside & 1886.0 & 45.1 \\
\hline 24 & Kaempferol 3-O-sophorotrioside & 718.9 & 7.2 \\
\hline 25 & Kaempferol 3-O-(sinapoyl)-sophoroside & 1244.0 & 62.8 \\
\hline $26+27$ & Kaempferol 3-O-(feruloyl)-sophorotrioside + kaempferol 3-O-(feruloyl)-sophoroside & 1988.8 & 33.1 \\
\hline 28 & Kaempferol 3-O-glucoside & $\begin{array}{l}\mathrm{nq} \\
\Sigma 13337.4\end{array}$ & - \\
\hline
\end{tabular}

${ }^{a}$ Results are expressed as mean of three determinations. SD standard deviation, $\sum$, sum of the determined phenolic compounds 


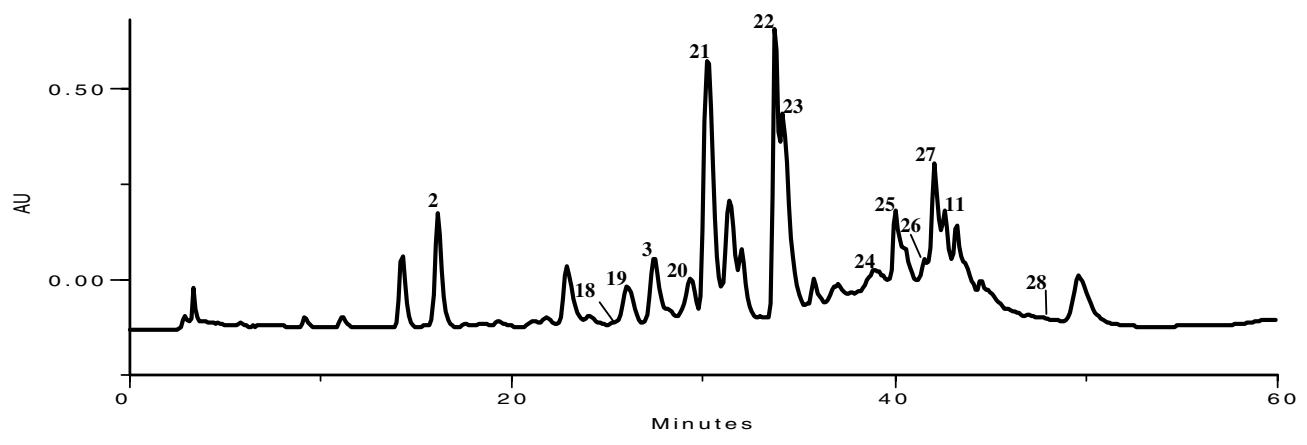

Fig. 9 HPLC-DAD phenolic profile of tronchuda cabbage external leaves hot-water extract. Detection at $330 \mathrm{~nm}$. Peaks: (2) 3-p-coumaroylquinic acid; (3) kaempferol 3-Osophoroside-7- $O$-glucoside; (11) kaempferol 3- $O$-sophoroside; (18) kaempferol 3-O-sophorotrioside-7- $O$-glucoside; (19) kaempferol 3-O-(methoxycaffeoyl/caffeoyl)-sophoroside-7- $O$-glucoside; $\quad(20)$ kaempferol 3-O-sophorotrioside-7- $O$-sophoroside; (21) kaempferol

displayed a concentration-dependent antioxidant potential, measured by the DPPH assay $\left(\mathrm{IC}_{25}\right.$ at $1192 \mu \mathrm{g} \mathrm{ml} \mathrm{m}^{-1}$ ), although with less effectiveness than external leaves $\left(\mathrm{IC}_{25}\right.$ at $440 \mu \mathrm{g} \mathrm{ml}^{-1}$ ) (Fig. 5).

Tronchuda cabbage internal leaves scavenged X/XOgenerated superoxide radical in a concentration dependent manner (Fig. 6A), with an $\mathrm{IC}_{50}$ at $351 \mu \mathrm{g} \mathrm{ml}^{-1}$, but its external leaves exhibited stronger antiradical capacity $\left(\mathrm{IC}_{50}\right.$ at $102 \mu \mathrm{g} \mathrm{ml}^{-1}$ ) (Fig. 6B). Since an inhibitory effect on the enzyme itself would also lead to a decrease in NBT reduction [31], the effect of the lyophilised extracts on the metabolic conversion of xanthine to uric acid was checked. The results demonstrate that the internal leaves extract had a weak inhibitory effect on XO, which was concentration dependent $\left(\mathrm{IC}_{10}\right.$ at $273 \mu \mathrm{g} \mathrm{ml}^{-1}$ ) (Fig. 6A), while external leaves had no effect on the enzyme (Fig. 6B). The capacity of both lyophilized extracts to scavenge superoxide radicals in a concentration-dependent way was confirmed when this radical was generated by a chemical system composed by PMS, NADH and oxygen. The results indicated that internal leaves, with an $\mathrm{IC}_{25}$ at $101 \mu \mathrm{g} \mathrm{ml}^{-1}$ (Fig. 6A), had lower ability to scavenge superoxide radical than external leaves $\left(\mathrm{IC}_{25}\right.$ at $43 \mu \mathrm{g} \mathrm{ml}^{-1}$ ) (Fig. 6B). So, the antioxidant activity exhibited by the internal leaves in the enzymatic assay is achieved by their capacity to act as both superoxide radical scavengers and $\mathrm{XO}$ inhibitors.

Tronchuda cabbage internal leaves extract also exhibited a potent scavenging activity for hydroxyl radical in a concentration dependent manner (Fig. 7A), with an $\mathrm{IC}_{25}$ at $27 \mu \mathrm{g} / \mathrm{ml}$, although less efficient than external leaves extract $\left(\mathrm{IC}_{25}\right.$ at $\left.10 \mu \mathrm{g} / \mathrm{ml}\right)$ (Fig. $\left.7 \mathrm{~B}\right)$. If we omit ascorbate in the reaction mixture, and if prooxidant compounds are present, they will be able to redox cycle the metal ion required for hydroxyl generation, and thus increase the radical production [40]. In order to evaluate the pro-oxidant potential of the extracts, we omitted ascorbic acid, and we found that both lyophilised extracts were not effective substitutes for ascorbic acid (Fig. 7). Thus, tronchuda cabbage internal and external leaves do not act as pro-oxidants. Some compounds inhibit deoxyribose degradation in this
3-O-sophoroside-7- $O$-sophoroside; (22) kaempferol 3-O(sinapoyl/caffeoyl)-sophoroside-7-O-glucoside; (23) kaempferol 3$O$ - (feruloyl/caffeoyl)-sophoroside-7-O-glucoside; (24) kaempferol 3-O-sophorotrioside; (25) kaempferol 3-O-(sinapoyl)-sophoroside; (26) kaempferol 3-O-(feruloyl)-sophorotrioside; (27); kaempferol 3-O-(feruloyl)-sophoroside; (28) kaempferol 3- $O$-glucoside

assay, not by reacting with hydroxyl radicals, but because they present ion-binding capacity and can withdraw the iron ions and render them inactive or poorly active in Fenton reactions [41]. Attending to this fact, the assay was also performed in the absence of EDTA in order to check the ability of the extracts to chelate iron ions. In the assay performed under these conditions both internal and external leaves lyophilised extracts had a similar behaviour, exhibiting a weak ability to chelate iron ions (Fig. 7).

The oxidizing properties of $\mathrm{HOCl}$ induce the conversion of TNB $\left(\lambda_{\max }=412 \mathrm{~nm}\right)$ to DTNB $\left(\lambda_{\max }=315 \mathrm{~nm}\right)$. In the present assay a $\mathrm{HOCl}$ scavenger inhibits the oxidation of TNB into DTNB [42]. Tronchuda cabbage internal leaves lyophilized extract exhibited a weak $\mathrm{HOCl}$ scavenging activity, in a concentration-dependent manner, as shown in Fig. 8. The external leaves revealed to have higher antioxidant capacity against $\mathrm{HOCl}$ (Fig. 8).

Despite the antioxidant capacity exhibited by tronchuda cabbage internal leaves, in general terms, and according to the results obtained in all assays, they exhibited lower antioxidant potential than external leaves. This can be ascribed to the higher content of both phenolics and organic acids in the external leaves (Tables 5 and 6), which are known to have antioxidant activity [43]. In addition, the qualitative phenolic composition is also different: in the external leaves extract, with the exceptions of 3-p-coumaroylquinic acid (2), kaempferol 3-Osophoroside-7- $O$-glucoside (3) and kaempferol 3-Osophoroside (11), several phenolic compounds were identified, namely flavonol glycosides, distinct from those detected in the internal leaves (Fig. 9). Either flavonol glycosides [44, 45] or hydroxycinnamic esters [46] have demonstrated antioxidant activity. However, as the content of flavonol glycosides is higher in external leaves than in internal ones (95 and 54\% of total phenolic compounds, respectively), this class of phenolics may contribute the most for the effects observed. On the other hand, the higher amount of acylated flavonols, namely caffeoyl derivatives, in the external leaves (Table 6) might also explain their potent antioxidant capacity. These compounds are reported 
to have high scavenging ability due to the presence of an $o$-dihydroxy structure in the caffeoyl moiety, which confers great stability to the radical form and participates in the electron delocalisation [44].

Concerning the organic acids, despite the presence of the previously mentioned seven compounds (Fig. 4), quantitative differences were noticed between internal and external leaves (Table 5), which may influence the antioxidant potential. Citric acid was the main compound in the external leaves, corresponding to $43 \%$ of total identified acids, followed by the pair malic plus quinic acids (28\%), while internal leaves presented ascorbic acid and the pair malic plus quinic acids in the highest amount (34\%, each). Thus, it seems that citric acid may have an important role in the antioxidant potential of tronchuda cabbage, as can be seen by the higher capacity exhibited by the external leaves. In fact, citric acid is known to protect ascorbic acid from metal-catalysed oxidation and to function as a synergist with other antioxidants [43].

In conclusion, the results obtained in the present work denote that tronchuda cabbage internal leaves may constitute a good source of health promoting compounds, namely flavonoids and organic acids. In addition, and as far as we know, this is the first report considering the antioxidant potential of the species, suggesting that it could be useful in the prevention of diseases in which free radicals are implicated.

Acknowledgements The authors are grateful to Fundação para a Ciência e Tecnologia (POCI/AGR/57399/2004) for financial support of this work. Vendula Vrchovská is grateful to European Union Erasmus/Socrates II Programme for a grant (MSM 002162 0822).

\section{References}

1. Kähkönen MP, Hopia AI, Vuorela HJ, Rauha JP, Pihlaja K, Kujala TS, Heinonen M (1999) J Agric Food Chem 47:39543962

2. Vinson JA, Hao Y, Su X, Zubik L (1998) J Agric Food Chem 46:3630-3634

3. Chu YF, Sun J, Wu X, Liu RH (2002) J Agric Food Chem 50:6910-6916

4. Pulido R, Bravo L, Saura-Calixto F (2000) J Agric Food Chem 48:3396-3402

5. Gyamfi MA, Yonamine M, Aniya Y (1999) Gen Pharmacol 32:661-667

6. Behl C, Moosmann B (2002) Free Radic Biol Med 33:182-191

7. du Toit R, Volsteedt Y, Apostolides Z (2001) Toxicol 166:63-69

8. Ninfali P, Bacchiocca M (2003) J Agric Food Chem 51:22222226

9. Lin CH, Chang CY (2005) Food Chem 90:9-15

10. Halliwell B, Aeschbach R, Löliger J, Aruoma OI (1995) Food Chem Toxicol 33:601-617

11. Sies H (1993) Eur J Biochem 215:213-219

12. Tseng TH, Kao ES, Chu CY, Chou FP, Lin Wu HW, Wang CJ (1997) Food Chem Toxicol 35:1159-1164
13. Liu RH (2004) J Nutr 134:3479S-3485S

14. Chun OK, Smith N, Sakagawa A, Lee CY (2004) Int J Food Sci Nutr 55:191-199

15. Beecher CWW (1994) Am J Clin Nutr 59:1166-1170

16. Stoewsand GS (1995) Food Chem Toxicol 33:1537-543

17. Park EJ, Pezzuto JM (2002) Cancer Metast Rev 21:231-255

18. Hertog MGL, Hollman PC, Van de Putte B (1993) J Agric Food Chem 41:1242-1246

19. Hollman PC, Hertog MGL, Katan MB (1996) Biochem Soc Trans 24:785-789

20. Galati G, O'Brien PJ, (2004) Free Radic Biol Med 37:28730

21. Llorach L, Espín JC, Tomás-Barberán FA, Ferreres F (2003) J Agric Food Chem 51:2181-2187

22. Proteggente AR, Pannala AS, Paganga G, van Buren L, Wagner E, Wiseman S, van De Put F, Dacombe C, Rice-Evans CA (2002) Free Radic Res 36:217-233

23. Kurilich AC, Jeffery EH, Juvik JA, Wallig MA, Klein BP (2002) J Agric Food Chem 50:5053-5057

24. Racchi M, Daglia M, Lanni C, Papetti A, Govoni S, Gazzani G (2002) J Agric Food Chem 50:1272-1277

25. Ferreres F, Valentão P, Llorach R, Pinheiro C, Cardoso L, Pereira JA, Sousa C, Seabra RM, Andrade PB (2005) J Agric Food Chem 53:2901-2907

26. Silva BM, Andrade PB, Mendes GC, Seabra RM, Ferreira MA (2002) J Agric Food Chem 50:2313-2317

27. Ferreres F, Llorach R, Gil-Izquierdo A (2004) J Mass Spectrom 39:312-321

28. Domon B, Costello A (1988) Glycoconj J 5:397-409

29. Hvattum E, Ekeberg D (2003) J Mass Spectrom 38:43-49

30. Silva BM, Andrade PB, Valentão P, Ferreres F, Seabra RM, Ferreira MA (2004) J Agric Food Chem 52:4705-4712

31. Valentão P, Fernandes E, Carvalho F, Andrade PB, Seabra RM, Bastos ML (2001) J Agric Food Chem 49:3476-3479

32. Valentão P, Fernandes E, Carvalho F, Andrade PB, Seabra RM, Bastos ML (2002) J Agric Food Chem 50:4989-4993

33. Llorach R, Gil-Izquierdo A, Ferreres F, Tomás-Barberán FA (2003) J Agric Food Chem 51:3895-3899

34. Vallejo F, Tomás-Barberán FA, Ferreres F (2004) J Chromatogr A 1054:181-193

35. Ferreres F, Llorach R, Gil-Izquierdo A (2004) J Mass Spectrom 39:312-321

36. Clifford MN, Johnston KL, Knight S, Kuhnert N (2003) J Agric Food Chem 51:2900-2911

37. Price KR, Casuscelli F, Colquhoun IJ, Rhodes MJC (1997) Phytochemistry 45:1683-1687

38. Vallejo F, Tomás-Barberán FA, Garcia-Viguera C (2003) Eur Food Res Technol 216:395-401

39. Fukumoto LR, Mazza G (2000) J Agric Food Chem 48:3597_ 3604

40. Li C, Xie B (2000) J Agric Food Chem 48:6362-6366

41. Payá M, Halliwell B, Hoult JRS (1992) Biochem Pharmacol 44:205-214

42. Künzel JKvFD, Zee Jvd, Ijzerman AP (1996) Drug Develop Res 37:48-54

43. Madhavi DL, Singhal RS, Kulkarni PR (1996) Natural antioxidants. In: Madhavi DL, Deshpande SS, Salunkhe DK (eds) Food antioxidants-Technological, toxicological and health perspectives. Marcel Dekker, New York, pp 73-76

44. Braca A, Fico G, Morelli I, De Simone F, Tomè F, De Tommasi N (2003) J Ethnopharmacol 86:63-67

45. Tang Y, Lou F, Wang J, Li Y, Zhuang S (2001) Phytochemistry 58:1251-1256

46. Plumb GW, Price KR, Rhodes MJ, Williamson G (1997) Free Radic Res 27:429-435 